\title{
Progression to tuberculosis disease increases with multiple exposures
}

\author{
Robyn S. Lee ${ }^{1,2,3}$, Jean-François Proulx ${ }^{4}$, Dick Menzies ${ }^{2,3,5}$ and \\ Marcel A. Behr ${ }^{1,2,3,6}$
}

Affiliations: ${ }^{1}$ Dept of Epidemiology, Biostatistics and Occupational Health, McGill University, Montreal, QC, Canada. ${ }^{2}$ McGill International TB Centre, Montreal, QC, Canada. ${ }^{3}$ The Research Institute of the McGill University Health Centre, Montreal, QC, Canada. ${ }^{4}$ Nunavik Regional Board of Health and Social Services, Kuujjuaq, QC, Canada. ${ }^{5}$ Respiratory Epidemiology and Clinical Research Unit, Montreal Chest Institute, Montreal, QC, Canada. ${ }^{6}$ Dept of Medicine, McGill University, Montreal, QC, Canada.

Correspondence: Robyn S. Lee, Research Institute of McGill University Health Centre, 1001 boul Décarie, Montréal, QC H4A 3J1 Canada. E-mail: robyn.s.c.leedgmail.com

ABSTRACT During a single year, a Canadian village had 34 individuals with microbiologically confirmed tuberculosis (TB) among 169 people with a new infection (20\%). A contact investigation revealed multiple exposures for each person. We investigated whether the intensity of exposure might contribute to this extraordinary risk of disease.

We carried out a case-control study using a public health database. Among those with a new infection, 34 had culture-confirmed TB (cases) and 118 did not progress to disease (controls). 17 patients with probable disease were excluded. Contact investigation data were utilised to tabulate the number of potential sources (total exposures). Generalised estimating equations with a logit link were used to identify associations between exposures and progression, and to investigate other potential risk factors.

The median (interquartile range) number of total exposures was 15 (3-23) for cases and 3 (2-12) for controls $(\mathrm{p}=0.001)$. The adjusted OR for disease was 1.11 (95\% CI 1.06-1.16) per additional exposure, corresponding to an OR of 3.4 for disease when comparing the medians of 15 versus 3 total exposures. This association increased when restricting to tuberculin skin test conversions.

Increased exposure could be a marker of greater risk of progression to TB disease. Therefore, this risk may not be transportable across epidemiologic settings with variable exposure intensities.

@ERSpublications

Increased exposure could not only be associated with risk of infection, but also risk of progression to TB disease http://ow.ly/aPCX302XC9j

This article has supplementary material available from erj.ersjournals.com

Received: May 042016 | Accepted after revision: Aug 022016 | First published online: Oct 062016

Support statement: This work was supported by the Canadian Institutes of Health Research (MOP number 125858, to D. Menzies and M.A. Behr). This funding agency had no role in the study design, data collection, analysis and interpretation of data, or in writing the manuscript or the decision to submit for publication. Funding information for this article has been deposited with the Open Funder Registry.

Conflict of interest: Disclosures can be found alongside this article at erj.ersjournals.com

Copyright OERS 2016 


\section{Introduction}

Between November 2011 and March 2012, there were 23 individuals diagnosed with tuberculosis (TB) in a Canadian village of only 933 people. In response to this crisis, public health and local clinical staff conducted extensive contact investigations of all persons diagnosed with active TB, to identify and treat those with prevalent disease, and to identify infected individuals at risk of progression. In all, 50 people were diagnosed with culture-confirmed TB by November 2012 (5\% of the village), including 34 of 169 newly infected contacts $(20 \%)$. This is in stark contrast to the $2-5 \%$ risk of progression that has previously been reported for the years immediately following TB infection [1-4]. Our previous studies on housing, nutrition and behavioural characteristics in this community $[5,6]$, which has a low prevalence of HIV, did not identify factors that could potentially account for this extraordinary rate of disease.

Public health data indicated that newly infected individuals had been in contact with multiple persons with active TB. Furthermore, molecular epidemiologic analysis revealed that what appeared at the level of the village to be a single "outbreak" was in fact the result of multiple contemporaneous transmission networks within the same community [7]. Given limited in- and out-migration, and the small size of the community, we could infer that many infected contacts had been repeatedly exposed. These observations led us to hypothesise that the intensity of exposure might explain the elevated risk of active TB disease. To investigate this possibility, we compared newly infected patients who progressed to disease with those who did not develop active TB.

\section{Materials and methods}

\section{Study design}

We conducted a case-control study using a public health database provided by the Nunavik Regional Board of Health and Social Services (NRBHSS). This database includes all individuals with active TB diagnosed in this community between November 2011 and November 2012 and their contacts.

\section{Active tuberculosis disease}

Individuals with $\geqslant 1$ culture positive for Mycobacterium tuberculosis were defined as having "confirmed TB". Culture results were assessed for potential cross-contamination as described in [7]. Persons with clinical and radiographic findings consistent with TB but without culture confirmation were classified as having "probable" TB.

\section{Contact investigation}

Persons with confirmed TB were interviewed upon diagnosis by trained healthcare providers to obtain lists of household and non-household contacts. Individuals were also asked about attendance at local "gathering houses", wherein public health suspected transmission might be occurring. These were homes of residents that also served as venues of socialisation, as there are no restaurants or bars in this community.

\section{Study inclusion criteria}

To assess the proximal risk of progression from infection to active $\mathrm{TB}$, we included only individuals with new TB infection.

\section{New infection}

A person was considered to have "new" TB infection if he/she had a positive tuberculin skin test (TST), either with no previous TST or with a previously documented negative TST (i.e. TST conversion).

\section{Cases and controls}

Individuals with new infection who had confirmed TB were included as cases. Contacts with new infection who did not progress to active TB in the year following infection were included as controls. A 1-year follow-up for progression was chosen based on two considerations: 1) the highest proportion of TB disease occurs in the first year following exposure $[2,8]$; and 2) because of renewed TB transmission in this village in 2014, we sought to avoid confusing 2011-2012 risk factors with those of the next wave of transmission.

\section{Exposure ascertainment}

To assess intensity of exposure, we examined the number of times an individual was listed as a contact of a potential source ("total exposures"). Clinical/demographic characteristics of these 50 potential source cases are provided in supplementary table S1. Each time a contact was listed by an individual with active $\mathrm{TB}$, this was counted as one exposure for the contact (i.e. this was done in a unidirectional fashion). This included household and non-household contact. Total exposures also included shared attendance or residence at gathering houses. The precise intensity of contact (i.e. duration and frequency) could not be 
included in modelling of exposure because these data were not obtained in a consistent manner throughout the outbreak. Additional details are provided in the supplementary material.

One person was never listed as a contact; this person was assigned one total exposure.

\section{Covariates}

Data were collected during the outbreak as part of routine contact investigation. Covariates were selected for inclusion from available data based on a priori consideration as determinants of TB. These included age at infection, sex, cigarette smoking, residing with a person with smear-positive disease, the number of persons per room (as a measure of housing occupancy), Bacillus Calmette-Guérin (BCG) vaccination and HIV/other comorbidities/immunosuppressive disorders. Precise definitions of each and how these were calculated (if applicable) have been provided in the supplementary material.

\section{Analysis}

The main analytic approaches and their respective sample sizes are outlined in figure 1 . In all analyses, the outcome of interest was progression to active TB disease. Our preliminary analysis (analysis 1a) included cases and controls as previously defined. Our secondary analysis (analysis 2a) restricted exposure to contact with individuals with smear-positive disease only, in order to assess whether the potential exposure-outcome relationship varied by smear status of potential sources.

We then performed several sensitivity analyses. First, contacts with a new positive TST who had not been previously tested may have already been positive before 2011-2012. To address this, we restricted the previous analyses to those with documented TST conversion (analysis $1 \mathrm{~b}$ and $2 \mathrm{~b}$ ).

Second, we repeated the above analyses (1ab, 2ab) using an alternative metric of exposure: the number of different genotypes to which an individual was exposed ("genotypic exposures"). Such exposures were tabulated based on contact investigation data. The genotypes of each individual with confirmed TB were previously identified in [7]. Notably, while there were sufficient genetic differences to assign the multiple chains of transmission, isolates were derived from a strain of $M$. tuberculosis first seen in this village in 2007, therefore it is unlikely that major differences in virulence had developed in the ensuing 6 years [7]. For further details on how these exposures were determined, please see the supplementary material.

Finally, because the public health response was amplified as of May 1, 2012, with extra clinical staff arriving to assist with contact investigations, we also assessed whether this change influenced results by stratifying analysis la by time.

Village $n=933$

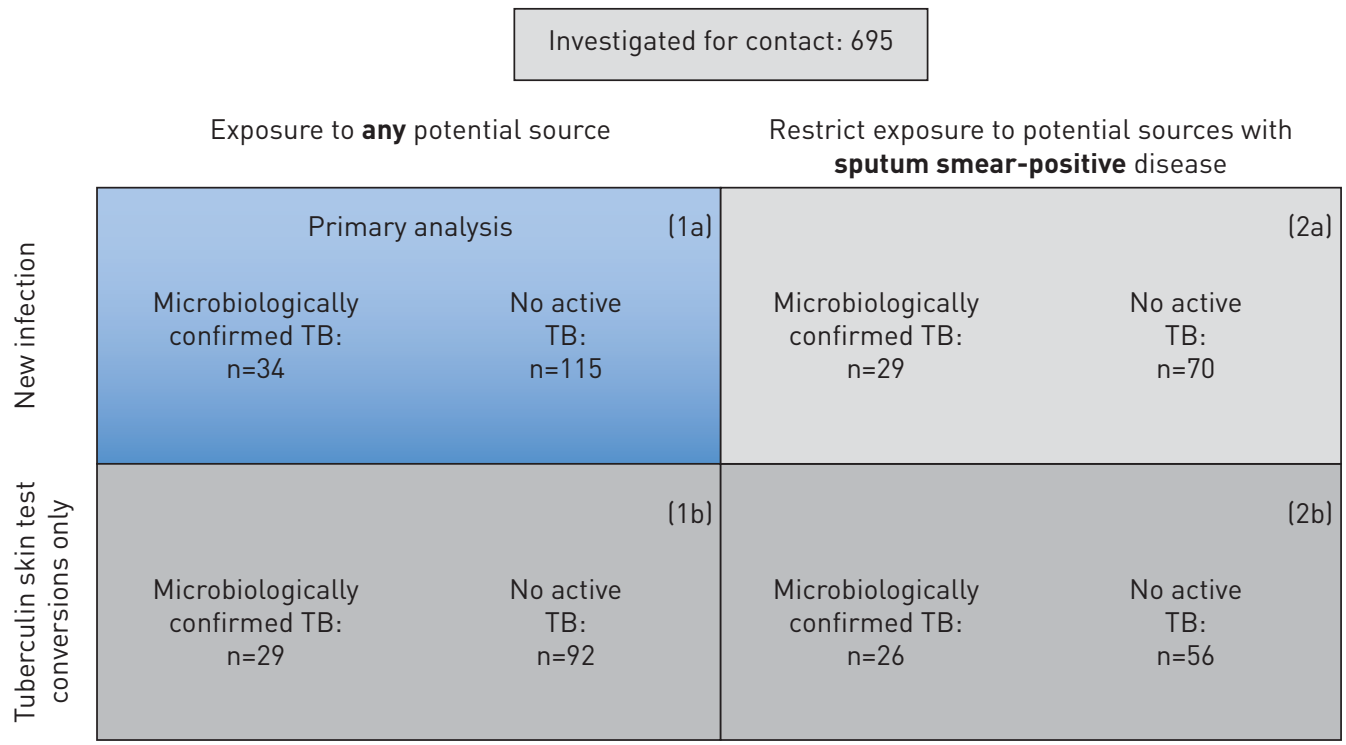

FIGURE 1 Main analytic approaches. The number of cases and controls included in each analysis are indicated. Note that three controls were excluded from the analyses owing to missing address. Fewer subjects are also present in analysis 2 as some individuals did not have contact with a smear-positive source. One person who did not have any reported contact was assigned a single total (and single genotypic) exposure as a minimum; this individual was not included in the smear-positive analysis as the contagiousness of his/ her potential source was unknown. TB: tuberculosis. 


\section{Statistical approaches}

Descriptive statistics were conducted and generalised estimating equations with a logit link were used to evaluate the association between potential risk factors and progression to TB disease, accounting for clustering by household. Multiple imputation with chained equations was used to estimate missing data (supplementary table S2). Variables with $\mathrm{p}<0.2$ on a univariate analysis were assessed in a multivariate analysis. Based on previously reported results [6], we looked for an association between residing with a person with smear-positive disease and the number of persons per room; in order to maintain hierarchy, both of these variables were included in preliminary multivariate models regardless of their significance in univariate analyses. Final models were selected using the quasi-information criterion (QICu) [9]. All analyses were conducted in Stata (v.13, StataCorp 2013).

\section{Ethics}

Ethics approval was obtained from the McGill University Faculty of Medicine Institutional Review Board and the NRBHSS. Individual patient consent was not required. All research was done in collaboration with the village council. Databases were linked and analysed in nominal form, under a professional mandate from the NRBHSS.

\section{Results}

Between November 2011 and November 2012, 695 of 933 villagers (74\%), including those with active TB, were investigated by the NRBHSS. Of 169 identified with new infection, 17 individuals were classified with "probable TB". These individuals presented distinct characteristics compared to those with confirmed disease (supplementary table S3) and controls (table 1), and thus they were excluded from analyses. Of the remaining 152 newly infected individuals, 34 had confirmed active TB - 31 with prevalent disease and three who developed disease within the year following identification of infection. Two of the latter had agreed to isoniazid prophylaxis, but did not complete therapy. All individuals had pulmonary TB. The remaining 118 individuals were classified as controls.

Summary characteristics of cases with confirmed TB and controls with new infection are shown in table 1 for analysis 1a. All additional analyses used subsets of these individuals. The address of residence was missing for three controls and these individuals were therefore excluded. Overall, cases and controls were similar in terms of age, sex, current cigarette smoking, BCG vaccination status and residing with a person with smear-positive disease $(p>0.05)$. No relevant comorbidities were identified in either cases or controls. Compared to controls, cases reported a higher number of total exposures $(\mathrm{p}=0.001)$ and resided in dwellings with higher occupancy, as measured by persons per room $(\mathrm{p}=0.036)$. Genotypic exposures were also higher for cases compared to controls $(\mathrm{p}=0.005)$.

Tables 2 and 3 show univariate and multivariate results for analyses 1 and 2, respectively. HIV and other comorbidities were not modelled owing to low/zero cell counts. All continuous variables had linear associations with the outcome $(\mathrm{p}>0.05)$, except for genotypic exposures in analysis 1a (supplementary table S4). The maximum number of total exposures to any potential source (analysis 1) was 28, while the maximum number of total exposures to sources with smear-positive disease only (analysis 2) was 8. Univariate

TABLE 1 Characteristics of individuals with exposure to any potential source, new infection (analysis 1a)

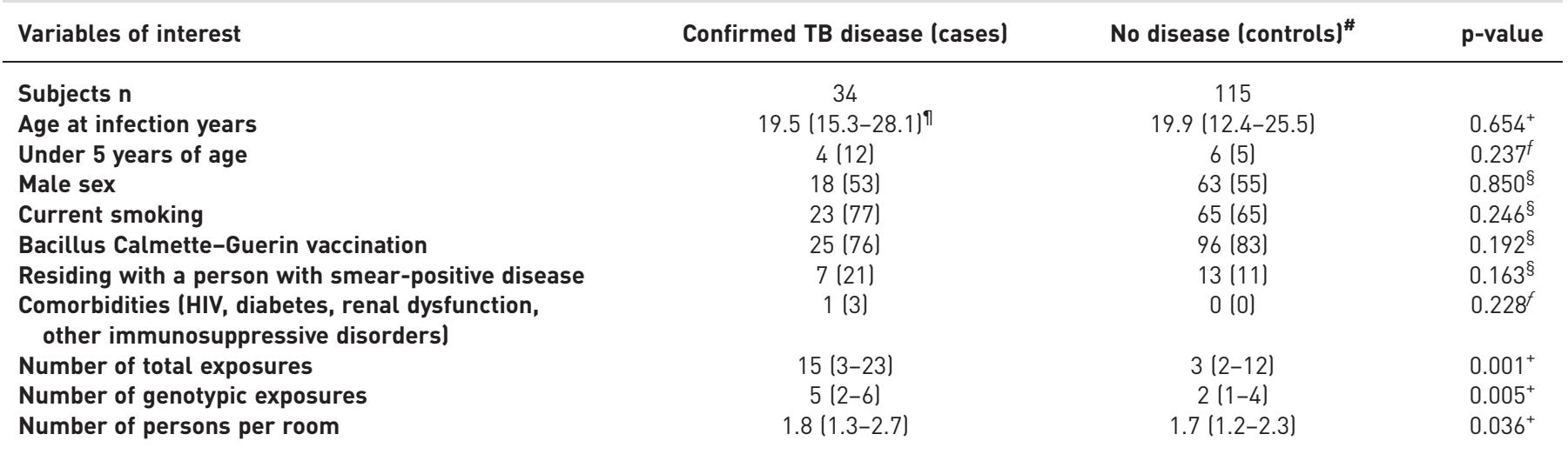

Data are presented as median (interquartile range) or $\mathrm{n}(\%)$, unless otherwise stated. TB: tuberculosis. \#: Three controls excluded from analysis as missing address of residence. ${ }^{1}$ : Age range for cases: 1.1-54.8 years, age range for controls: 0.5-59.4 years. ${ }^{+}$: Mann-Whitney rank sum test. ${ }^{\S}$ : Chi-squared test with 1 degree of freedom. ${ }^{f}$ : Fisher's exact test. Non-missing data are used for the denominator of proportions. A two-sided $p$-value of $<0.05$ is considered statistically significant. 
TABLE 2 Exposure to any potential source and progression to active tuberculosis

\begin{tabular}{|c|c|c|c|c|c|}
\hline & \multicolumn{3}{|c|}{ Univariate } & \multicolumn{2}{|c|}{ Multivariate } \\
\hline & OR & $95 \% \mathrm{Cl}$ & p-value & OR & $95 \% \mathrm{Cl}$ \\
\hline \multicolumn{6}{|l|}{ Analysis 1a - Contact with any potential source, newly diagnosed infection } \\
\hline Male sex & 0.93 & $0.44-1.94$ & 0.844 & Not in final model & \\
\hline Current smoking & 1.63 & $0.58-4.54$ & 0.351 & Not in final model & \\
\hline BCG & 0.65 & $0.24-1.75$ & 0.391 & Not in final model & \\
\hline Persons per room & 1.12 & $0.98-1.28$ & 0.086 & 1.18 & $1.04-1.34$ \\
\hline \multicolumn{6}{|l|}{$\begin{array}{l}\text { Analysis } 1 \mathrm{~b}-\text { Contact with any potential source, tuberculin skin } \\
\text { test conversion only }\end{array}$} \\
\hline Age at infection & 1.01 & $0.98-1.04$ & 0.593 & Not in final model & \\
\hline Male sex & 0.72 & $0.31-1.67$ & 0.442 & Not in final model & \\
\hline Current smoking & 2.19 & $0.61-7.90$ & 0.231 & Not in final model & \\
\hline $\begin{array}{l}\text { Persons per room }{ }^{\#} \text { when not residing with a person with smear-positive } \\
\text { disease }\end{array}$ & & & & 1.15 & $1.03-1.28$ \\
\hline $\begin{array}{l}\text { Persons per room }{ }^{\#} \text { when residing with a person with smear-positive } \\
\text { disease }\end{array}$ & & & & $1.49^{\pi}$ & $1.06-2.10$ \\
\hline \multicolumn{6}{|c|}{$\begin{array}{l}\text { BCG: Bacillus Calmette-Guérin. \#: For comparability to [6], persons per room scaled such that the odds ratio corresponds to a one-person } \\
\text { increase in a five-person house. ": } p=0.027 \text { for interaction between persons per room and residing with a person with smear-positive disease; } \\
\text { this odds ratio represents the joint effect of adding one person to a five-person house when residing with an individual with smear-positive } \\
\text { disease. Age and persons per room are centred at the overall mean for analysis } 1 \text { a, at } 20.8 \text { years and } 1.7 \text { persons per room, respectively. Total } \\
\text { exposures centred at } 1 \text {, as all individuals had at least one contact. }\end{array}$} \\
\hline
\end{tabular}

analysis showed a significant association between the number of total exposures and disease, irrespective of type of contact (analyses 1 and 2) or definition of new infection (analyses $1 \mathrm{~b}$ and 2b). Persons per room was also significantly associated with disease in both analyses.

TABLE 3 Exposure to potential sources with smear-positive disease only and progression to active tuberculosis

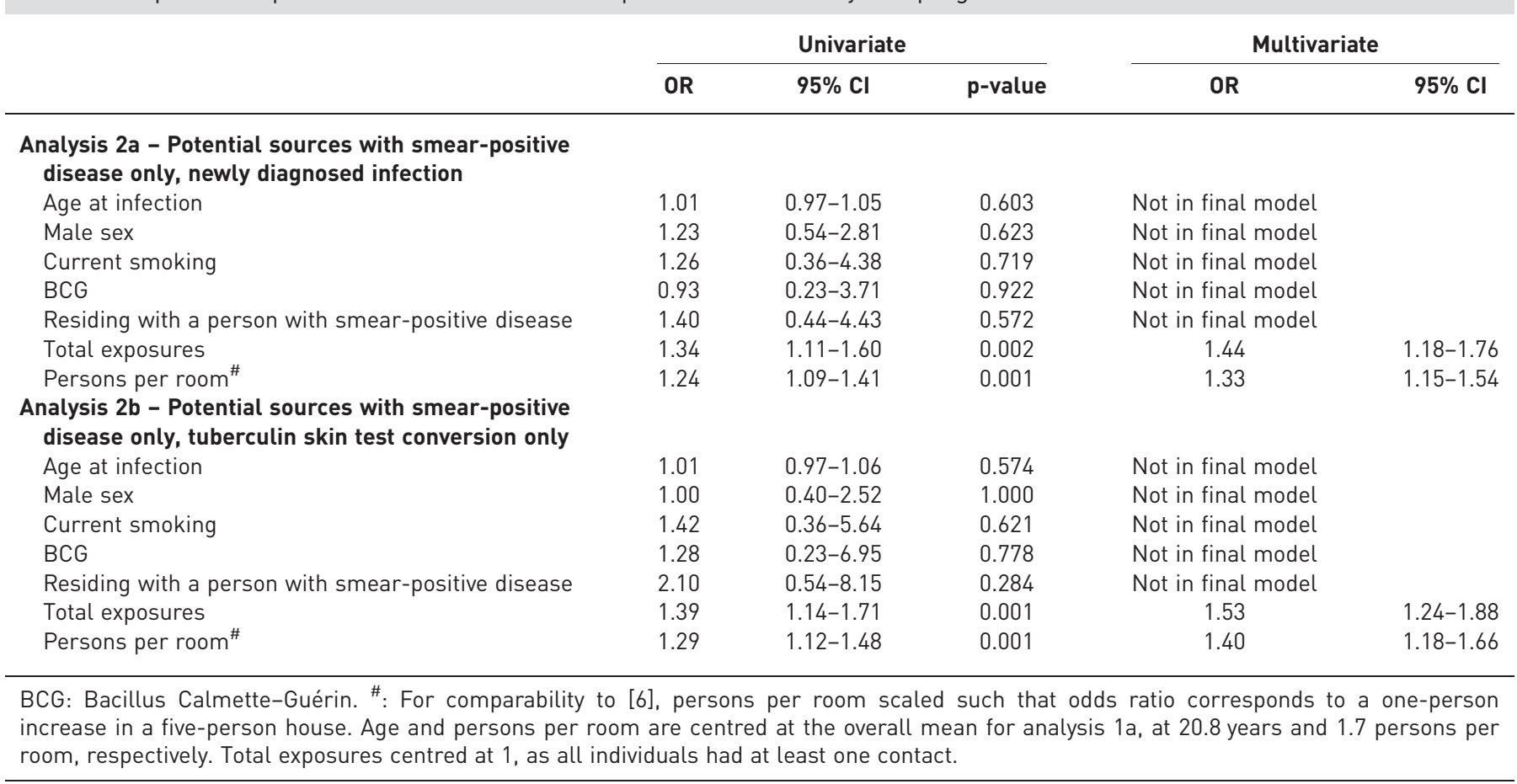


In all multivariate analyses (analyses $1 \mathrm{a}, 1 \mathrm{~b}, 2 \mathrm{a}$ and $2 \mathrm{~b}$ ), the number of total exposures was associated with progression to active TB disease, with adjusted ORs ranging from 1.11 to 1.53 for each one-person increase in contact (tables 2 and 3). Persons per room was also significantly associated with progression; the addition of one person to a five-room dwelling was associated with adjusted ORs from 1.18 to 1.40 (analyses 1a, 2a and 2b). When restricted to TST conversions (analysis 1b), an interaction between persons per room and residing with a smear-positive individual was detected; the odds of progression were higher with increased occupancy when a smear-positive individual lived in the same residence compared to houses without such individuals. However, given that the $95 \%$ confidence intervals overlap, this was inconclusive.

Similar results were obtained when the number of different genotypes was used instead as the exposure variable (supplementary tables S4 and S5). There were a maximum of seven genotypic exposures when considering contact with any potential source versus six genotypic exposures when analyses were restricted to smear-positive sources only.

To assess the potential influence of clinical staffing changes on May 1, 2012, we conducted separate analyses restricting to contact with persons diagnosed (and therefore interviewed) before or after this date. The numbers of exposures, and their association with progression to active TB, were similar across time periods (table 4).

\section{Discussion}

Our analysis revealed a significant association between the number of times an individual with recent infection was exposed to active TB and progression to disease. Adjusting for housing occupancy, we found the odds of disease were $\sim 1.1$-fold higher for each additional exposure, corresponding to an OR of 3.4 when comparing the median exposures actually experienced by individuals in this community. These results were consistent across all analyses, including when we imposed a more rigorous definition for infection, restricting to TST conversions, and when we restricted our exposure measurement to smear-positive sources only. These findings were also unaffected by changes in staffing during the outbreak.

TABLE 4 Exposure to any potential source and progression to active tuberculosis (analysis 1a), stratified by time of diagnosis of the potential source

Cases Controls

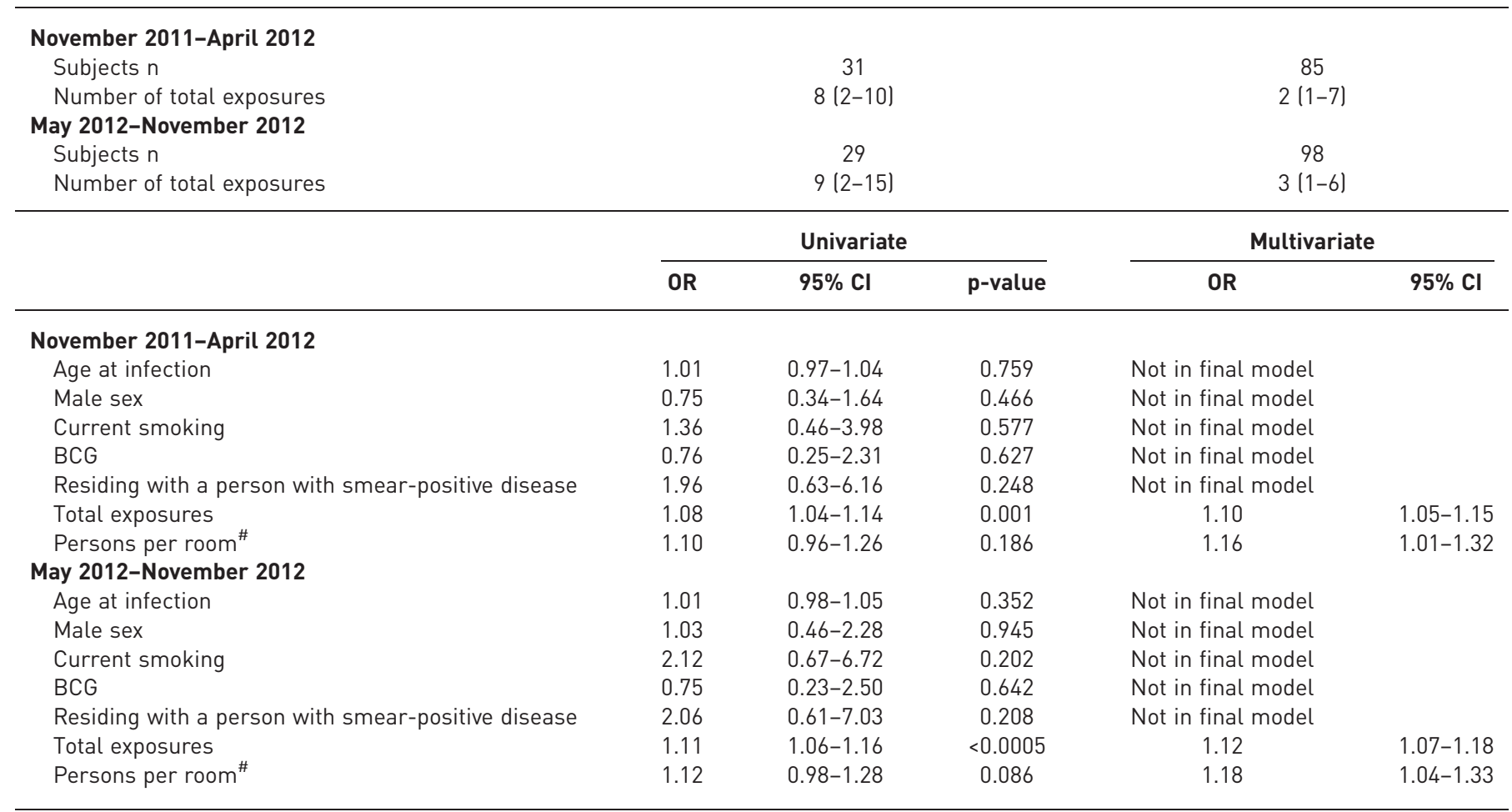

Data are presented as median (interquartile range), unless otherwise stated. BCG: Bacillus Calmette-Guérin. \#: For comparability to [6], persons per room scaled such that odds ratio corresponds to a one-person increase in a five-person house. Age and persons per room are centred at the overall mean for analysis $1 \mathrm{a}$, at 20.8 years and 1.7 persons per room, respectively. Total exposures centred at 1 , as all individuals had at least one contact. 
We propose two possible explanations for the observed association, which need not be mutually exclusive. A first possibility is that the number of exposures is a marker of increased probability of encountering a highly transmissible source. It has been proposed that $20 \%$ of all infectious disease cases are responsible for $80 \%$ of transmission [10], with the majority of cases either not transmitting at all or very minimally. Such "super-spreading" has been reviewed in [11] and reported for severe acute respiratory syndrome, Middle East respiratory syndrome and other pathogens. Anecdotal evidence [12, 13] and the high heterogeneity observed in cluster sizes on genotyping [14] suggest this phenomenon also occurs in TB. Our data were consistent with such an explanation, as there were highly contagious cases with smear-positive, cavitary disease within each subgroup of transmission (identified in [7]).

Another possible explanation is that repeated exposures directly influence progression. Increasing exposure (via inoculum size) has been shown to result in more extensive pathology in animals [15-17]; however, the role dose plays in the development of disease has not been fully elucidated. Experimental human challenges have not been done for TB, nor to our knowledge for other respiratory bacterial pathogens. In other infectious diseases, a dose response from infection to disease has been reported for Salmonella [18, 19], but not Campylobacter [20] or Cryptosporidium [21, 22]. To ethically investigate this in TB, observational data have been necessary. Previous studies (reviewed in [23]) relying on categorical measures of exposure, such as close versus casual contact [2, 24, 25], household versus non-household [26], or the nature of occupational exposure [27] have also supported an association with progression to disease.

Regardless of the mechanism, we propose that the number of exposures could serve as a useful marker of risk for progression in those with recent infection. Unlike exposure to a super-spreader, which is only known retrospectively following genotyping, the number of times a person is identified as a contact is tabulated in real-time during public health investigations. From a clinical and public health perspective, closer monitoring could be warranted for repeatedly exposed individuals as they could be at a higher risk of progressing to disease.

This study had a number of strengths. The dichotomous approach used previously to assess exposure can result in substantial residual confounding. In this study, we were able to obtain continuous metrics of exposure to quantify whether there is an association between increased exposure and risk of disease. Using contact investigation data collected in real-time and building on our molecular epidemiologic analyses [7], we tabulated the total number of exposures to different potential sources of TB, as well as the number of genotypic exposures. For both metrics, there were increased odds of progression from infection to disease with higher exposure. An additional strength was the limited out-migration from this region, which facilitated collection of complete contact investigation data and 100\% follow-up for progression to active TB. Finally, as most persons had resided in the village since birth, we also had access to complete, life-long medical records to assess for comorbidities and TB risk factors.

This study has several limitations. Sample size was limited by the extent of the public health crisis, with 34 individuals diagnosed with active TB among those with new infection during the study period. This could have reduced our power to detect associations between other covariates and progression to disease. Data were also collected as part of the public health response to the outbreak, rather than to test specific research hypotheses. As such, we could only assess variables routinely collected during a TB control investigation. Standardised contact investigation tools ensured that key covariates such as age and sex were consistently documented, however, and we note that follow-up studies in this village have similarly reported a lack of association between these covariates and progression to active TB [5, 6]. Unlike most environments where contact investigation has been studied, the epidemiologic context in this study was more homogeneous: $>90 \%$ of the individuals were Inuit [28], they resided in the same isolated Northern community and many of the social characteristics were similar, potentially reducing the ability to detect associations with disease. Smoking, for example, is quite prevalent in this community; without an exposure gradient, we could not detect an association in this context, despite smoking being linked to TB disease in many other populations. In accordance with the previous studies in this village, we found that housing occupancy was associated with progression, thereby strengthening confidence in our results. Finally, while the small size of the community and limited migration has made it feasible to detect all cases and subsequently perform large-scale contact investigations, this may not be as feasible in other settings with greater migration and loss to follow-up.

Through this analysis, we have shown that multiple exposures to TB are associated not only with increased infection, but also increased progression to disease. From a public health standpoint, such exposures could therefore serve as a marker of increased risk of progression to disease. Given the unique nature of this outbreak, these findings need to be validated in other settings. If exposure intensity is a marker of progression to TB disease, then attack rates from low-prevalence settings could under-estimate the risk of disease in settings where multiple exposures are more likely, and vice versa. 


\section{Acknowledgements}

The authors thank the village council and residents for their collaboration and engagement in this study. The authors also thank the staff of the Local Community Service Centre for their hard work during the outbreak, including collection of contact investigation data, and the Nunavik Regional Board of Health and Social Services for provision of clinical and epidemiologic data used in this study. The authors also thank David C. Alexander (Saskatchewan Disease Control Laboratory, Regina, SK, Canada), Brian Ward (Centre for the Study of Host Resistance, McGill University Health Centre, Montreal, QC, Canada) and Cedric Yansouni (Faculty of Medicine, McGill University, Montreal, QC, Canada), for sharing historical papers.

\section{References}

1 Mack U, Migliori GB, Sester M, et al. LTBI: latent tuberculosis infection or lasting immune responses to M. tuberculosis? A TBNET consensus statement. Eur Respir J 2009; 33: 956-973.

2 Sloot R, Schim van der Loeff MF, Kouw PM, et al. Risk of tuberculosis after recent exposure. A 10-year follow-up study of contacts in Amsterdam. Am J Respir Crit Care Med 2014; 190: 1044-1052.

3 Downes J. A study of the risk of attack among contacts in tuberculous families in a rural area. Am J Epi 1935; 22: 731-742.

4 Canadian Thoracic Society and Public Health Agency of Canada. Canadian Tuberculosis Standards. 7th Edn. 2014.

5 Fox GJ, Lee RS, Lucas M, et al. Inadequate diet is associated with acquiring Mycobacterium tuberculosis infection in an Inuit community: a case-control study. Annals Am Thorac Soc 2015; 12: 1153-1162.

6 Ahmed Khan F, Fox GJ, Lee RS, et al. Housing characteristics as determinants of tuberculosis in an Inuit community: a case-control study. 19th Annual Conference of The International Union Against Tuberculosis and Lung Disease - North America Region. 26-28 February 2015. Vancouver, Canada; 2015.

7 Lee RS, Radomski N, Proulx J-F, et al. Reemergence and amplification of tuberculosis in the Canadian arctic. J Infect Dis 2015; 211: 1905-1914.

8 Fox GJ, Barry SE, Britton WJ, et al. Contact investigation for tuberculosis: a systematic review and meta-analysis. Eur Respir J 2013; 41: 140-156.

9 Pan W. Akaike's information criterion in generalized estimating equations. Biometrics 2001; 57: 120-125.

10 Woolhouse ME, Dye C, Etard JF, et al. Heterogeneities in the transmission of infectious agents: implications for the design of control programs. Proc Natl Acad Sci USA 1997; 94: 338-342.

11 Lloyd-Smith JO, Schreiber SJ, Kopp PE, et al. Superspreading and the effect of individual variation on disease emergence. Nature 2005; 438: 355-359.

12 Kiers A, Drost AP, van Soolingen D, et al. Use of DNA fingerprinting in international source case finding during a large outbreak of tuberculosis in The Netherlands. Int J Tuberc Lung Dis 1997; 1: 239-245.

13 Kline SE, Hedemark LL, Davies SF. Outbreak of tuberculosis among regular patrons of a neighborhood bar. N Engl J Med 1995; 333: 222-227.

14 Ypma RJF, Altes HK, van Soolingen D, et al. A sign of superspreading in tuberculosis. Epidemiology 2013; 24 395-400.

15 Koch R, Codell Carter K. Essays of Robert Koch. Connecticut, Greenwood Press, 1987.

16 Ratcliffe HL. Tuberculosis induced by droplet nuclei infection. Am J Epidemiol 1952; 55: 36-48.

17 Perla D. Experimental epidemiology of tuberculosis. J Exp Med 1927; 45: 209-226.

18 McCullough N, Eisele W. Experimental human salmonellosis. I. Pathogenicity of strains of Salmonella Meleagridis and Salmonella Anatum obtained from spray-dried whole egg. J Infect Dis 1951; 88: 278-289.

19 Blaser MJ, Newman LS. A Review of human salmonellosis: I. Infective dose. Rev Infect Dis 1982; 4: 1096-1105.

20 Black RE, Levine MM, Clements ML, et al. Experimental Campylobacter jejuni infection in humans. J Infect Dis 1988; 157: 472-479.

21 Chappell CL, Okhuysen PC, Langer-Curry RC, et al. Cryptosporidium muris: infectivity and illness in healthy adult volunteers. Am J Trop Med Hyg 2015; 92: 50-55.

22 DuPont HL, Chappell CL, Sterling CR, et al. The infectivity of Cryptosporidium parvum in healthy volunteers. N Engl J Med 1995; 332: 855-859.

23 Salgame P, Geadas C, Collins L, et al. Latent tuberculosis infection - revisiting and revising concepts. Tuberculosis (Edinb) 2015; 95: 373-384.

24 Grzybowski S, Barnett GD, Styblo K. Contacts of cases of active pulmonary tuberculosis. Bull Int Union Tuberc Lung Dis 1975; 50: 90-106.

25 Houk VN, Baker JH, Sorensen K, et al. The epidemiology of tuberculosis infection in a closed environment. Arch Environ Health 1968; 16: 26-35.

26 Moran-Mendoza O, Marion SA, Elwood K, et al. Risk factors for developing tuberculosis: a 12-year follow-up of contacts of tuberculosis cases. Int J Tuberc Lung Dis 2010; 14: 1112-1119.

27 Ferguson RG. BCG vaccination in hospitals and sanatoria of Saskatchewan; a study carried out by the Nationa Research Council of Canada. Can J Public Health 1946; 37: 435-451.

28 Statistics Canada. Kangiqsualujjuaq, Quebec (Code 2499090). National Household survey. Statistics Canada catalogue no. 99-011-X20110007. www12.statcan.gc.ca/nhs-enm/2011/dp-pd/aprof/index.cfm?Lang=E. Date last updated: November 27, 2015. Date last accessed: May 4, 2016. 